\title{
Speckle tracking echocardiography derived systolic longitudinal strain is better than rest single photon emission tomography perfusion imaging for nonviable myocardium identification
}

\author{
Martin Hutyra ${ }^{a}$, Tomas Skala ${ }^{a}$, Milan Kaminek ${ }^{b}$, David Horakc, Martin Kocherc, Zbynek Tudosc, Jiri Jarkovsky d, Jan Preceka, \\ Milos Taborsky ${ }^{a}$
}

\begin{abstract}
Background. The aim was to compare the speckle tracking echocardiography (STE) derived systolic longitudinal strain $\left(\mathrm{SL}_{\text {Smax }}\right)$ with rest single photon emission computed tomography (SPECT) perfusion imaging $\left(\mathrm{Q}_{\mathrm{REST}}\right)$, and to define the optimal cut-offs for $\mathrm{SL}_{\text {smax }}$ to discriminate transmural scar on contrast-enhanced magnetic resonance imaging (ceCMR). Methods and Results. In 100 patients with chronic ischemic left ventricular (LV) dysfunction, myocardial viability was assessed using STE and rest SPECT to predict LV segmental relative extent of delayed enhancement (DE) $>75 \%$ on ceCMR. Correlation was found between regional $\mathrm{SL}_{\mathrm{Smax}_{\max }}(\mathrm{r}=-0.59, P<0.0001)$ and $\mathrm{DE}$ on ceCMR. The $\mathrm{SL}_{\mathrm{Smax}_{\max }}$ optimal cutoff $-5.3 \%$ identified segments with DE $>75 \%$ on ceCMR (sensitivity $83.1 \%$, specificity $84.6 \%$ ). Optimal cut-offs $\mathrm{SL}_{\text {Smax }}$ for segments corresponding to individual perfusion territories $(-3.6 \%,-5.3 \%$ and $-4.7 \%$ for $L A D, L C x$ resp. RCA perfusion territories) were identified. There was a significant difference (AUC 0.866 vs. 0.822 for $S L_{\text {Smax }}$ resp. $Q_{R E S T^{\prime}} p=0.036$ ) in the accuracy of predicting non-viable segment due to the greater accuracy of $\mathrm{SL}_{\text {Smax }}$ than $\mathrm{Q}_{\mathrm{REST}}$ in the RCA perfusion territory (AUC 0.893 vs. 0.75 for $\mathrm{SL}_{\text {Smax }}$ resp. $Q_{\text {REST }} P=0.001$ ).

Conclusions. STE enabled identification of LV non-viable segments. Cut-off values derived for perfusion territories of individual coronary arteries improve the accuracy of predicting a transmural scar presence. In comparison with rest myocardial SPECT perfusion imaging, STE is more accurate in predicting non-viable myocardium.
\end{abstract}

Key words: myocardial viability, speckle tracking echocardiography, two-dimensional strain, rest myocardial perfusion imaging, cardiac magnetic resonance imaging, delayed enhancement

Received: February 27, 2012; Accepted with revision: July 9, 2012; Available online: September 5, 2012

http://dx.doi.org/10.5507/bp.2012.072

${ }^{a}$ Department of Internal Medicine I - Cardiology, University Hospital Olomouc, Czech Republic

${ }^{b}$ Department of Nuclear Medicine, University Hospital Olomouc

'Department of Radiology, University Hospital Olomouc

dInstitute of Biostatistics and Analyses, Faculty of Medicine and the Faculty of Science, Masaryk University Brno

Corresponding author: Tomas Skala, e-mail: tomasskala@gmail.com

\section{INTRODUCTION}

Ischemic cardiomyopathy is the most frequent cause of LV systolic dysfunction with the heart failure syndrome ${ }^{1}$. Revascularization in patients with coronary artery disease and left ventricular (LV) dysfunction has been shown to improve LV function, heart failure symptoms and prognosis $^{2,3}$. Unfortunately, only a percentage of revascularized patients show $L V$ reverse remodeling. For this reason, preprocedural identification of the ratio between viable and non-viable myocardium is necessary.

Contrast enhanced cardiac magnetic resonance (ceCMR) is considered as a reference imaging method in the diagnostics of myocardial viability in chronic ischemic heart disease. It has shown close correlation between the extent of delayed enhancement and myocardial scar size. CeCMR has a high accuracy in the identification of reversible myocardial dysfunction before revascularization ${ }^{4}$. Single photon emission computed tomography (SPECT) is considered to be a powerful predictor of myocardial viability $^{5}$. CeCMR based parameters of segmental scar transmurality have greater accuracy in predicting reverse
LV remodeling than rest SPECT results in predicting long term LV reverse remodeling and LV ejection fraction improvement after surgical revascularization ${ }^{6}$. Speckle tracking echocardiography (STE) has become a simple, rapid, relatively accurate and angle-independent echocardiographic method to assess and quantify regional myocardial function based on tracking of specific speckle patterns created by interference of ultrasound beams in the myocardium. Myocardial STE derived strain measurement has been validated, using sonomicrometry and tagged CMR as reference methods ${ }^{7}$. In individuals with chronic ischemic heart disease, STE derived parameters correlate with the extent of affected coronary arteries, predict the recovery of the LV function after surgical revascularization, and are capable of differentiating potentially viable segments from those showing scar extent above $50 \%\left(\right.$ ref. $\left.^{8-10}\right)$. However, these segments can display even more than 30 percent of the functional recovery after revascularization ${ }^{11}$.

The aim of this study was direct comparison between the myocardial deformation analysis and rest perfusion imaging (cardiac gated SPECT) in predicting LV seg- 
mental scar transmurality over $75 \%$ thickness of the LV segment wall, which is viewed as a correlative to an unequivocally non-viable tissue with no possibility of functional recovery after myocardium revascularization ${ }^{4}$. This study also sought to define, in patients with ischaemic left ventricular dysfunction, whether the STE and SPECT accuracy in detecting a transmural scar is comparable with the area of the coronary circulation in the LV frontal, lateral, and inferior myocardial wall.

\section{METHODS}

\section{Patient group}

We consecutively enrolled 100 patients admitted to our department in the period from March 2005 to June 2009 with ischemic cardiomyopathy and LV ejection fraction $<40 \%$ on 2D echocardiography (biplane Simpson formula). The patients were candidates for myocardial revascularization based on the coronary angiography findings. All had stable LV dysfunction but those who experienced myocardial infarction during the last 6 months prior to admission were excluded. Patients with acute coronary syndromes or any signs of acute myocardial ischemia as well as patients with significant valvular disease, atrial fibrillation or contraindications for ceCMR (claustrophobia, implanted pacemaker or ICD) were also excluded. All patients signed informed consent and the study protocol was approved by the local ethics committee. Within one week after coronary angiography patients underwent echocardiography, ceCMR and SPECT.

\section{Speckle tracking echocardiography}

Echocardiographic examinations were performed on the ultrasound Vivid 7 (GE Healthcare Technologies, Waukesha, Wisconsin, USA) equipped with an M3 ultrasound probe $(3.5 \mathrm{MHz})$ with the possibility of a complete full-fledged analysis on archived sequences. The patients were examined in the left lateral decubitus position in respiration at rest, and the registered sequences for the follow-up evaluation were obtained in a calm exhalation to minimize translational motions of the heart. In compliance with the stated recommendations, the standardized 17-segment LV model was used for a detailed evaluation of the LV segments by all the applied methods ${ }^{12}$. The resting examination with an obligatory synchronous registration of 1 ECG lead was performed standardly from parasternal projections on LV long and short axis and from apical long axis, 2 and 4 chamber projections. In order to set an adequate timing for the beginning and end of a systolic ejection phase, the flow in the left ventricular outflow tract was registered, and subsequently the beginning (aortic valve opening - AVO) and end (aortic valve closure - AVC) of the LV ejection phase were indicated. From the above projections, we registered the B-mode second harmonic sequence with a frame rate setting optimized for the specific method (optimal frame rate $70 \mathrm{~s}^{-1}$, range between $40-100 \mathrm{~s}^{-1}$ ). The evaluation of echocardiographic findings was performed off-line in an environment of ar- chival program EchoPac 7 Option (version BT 10.0.0) by two independent physicians blindly without knowledge of clinical status or results of other examinations. While making the off-line analysis, in individual projections the LV endocardial surface was tracked, together with search for the region of interest (ROI) and its follow-up adjustment for the displayed left ventricle of the specific projection. The software divided the LV into individual segments for the specific projection and made subsequent semiautomatic quantitative spectral analysis with the possibility of manual corrections. The STE derived peak systolic longitudinal strain $\left(\mathrm{SL}_{\mathrm{Smax}}\right)$ was the observed parameter. Intraobserver and interobserver agreement in the analysis of myocardial deformation parameters were determined in 10 subjects (160 LV segments). For intraobserver agreement, the Lin coefficient for $\mathrm{SL}_{\mathrm{Smax}}$ was 0.98 (95\% CI 0.96 to 0.99 ), and Pearson correlation coefficient was 0.95 . For interobserver agreement, the Lin coefficient for SLs was 0.95 (95\% CI 0.93 to 0.96 ) and Pearson correlation coefficient was 0.92 for $\mathrm{SL}_{\mathrm{Smax}}$.

\section{Magnetic resonance imaging}

The magnetic resonance examinations were performed on 1.5T Magnetom Avanto (Siemens Medical Solutions, Erlangen, Germany), Q engine (33 mT/m), Tim 76x18, equipped with software Syngo, advanced cardiac package and post processing software Argus. The standard myocardial viability evaluation protocol comprised a functional cinematic examination focused on LV using TrueFisp2DCINE sequence (assessment of LV end-diastolic thickness and EF) and delayed enhancement (DE) depiction using high space-resolution sequences CE-IR-MRI. Both types of ceCMR evaluation of LV were performed using a 5-element phased-array cardiac coil with the patient placed supine in identical projections (series of layers in short axis, 4-chamber, 2-chamber and LV outflow tract projections). Typical parameters for trueFisp2D-CINE sequences were: matrix 192x156, FOV 366×297 mm, voxel size 1.9x1.9x6 mm, slice thickness $6 \mathrm{~mm}$, TR/TE/FA 41.72 $\mathrm{ms} / 1.25 \mathrm{~ms} / 74 \mathrm{deg}$., 14 segments, phase $41.52 \mathrm{~ms}$, total acquisition time $1.16 \mathrm{~min}$. (TA), 9 slices, 9 breath-hold (BH), PAT factor 2, ECG retrospective gating, calculated 25 phases, bandwidth (BW) $930 \mathrm{~Hz} / \mathrm{Px}$. To assess DE a paramagnetic extracellular contrast agent (CA) based on gadolinium (1 molar Gadovist, Bayer Schering Pharma, Berlin, Germany) was applied intravenously dosed 0.15 $\mathrm{mL} / \mathrm{kg}$. Images were acquired during breath-holds. The scan started 15 min after CA application. Phase Sensitive Inversion Recovery (PSIR) sequence without a need for manual adjustment of inversion time (TI) to null the viable myocardium signal was used. Typical parameters for PSIR sequences were: matrix 256x256, FOV 400x275 $\mathrm{mm}$, voxel size $2.2 \times 1.6 \times 8 \mathrm{~mm}$, slice thickness $5 \mathrm{~mm}, \mathrm{TR} /$ TE/FA 700 ms/1.35 ms/45 deg., non-selective IR, TI 300 ms, 65 segments, TA 5, BH, PAT factor 1, ECG trigger, 2nd trigger pulse, BW $780 \mathrm{~Hz} / \mathrm{Px}$. Automatically reconstructed pictures of this sequence were used to assess myocardial viability. Every LV segment was scanned for the extent and transmurality of scar after MI: maximal 
and average scar width in each segment $(\mathrm{mm})$, average $\mathrm{LV}$ wall width in each segment $(\mathrm{mm})$ and average scar extent in each segment (\%). The segmental extent of delayed enhancement (DE) was calculated, defined as the percentage contrast-enhanced area of the total myocardial area $\left[\right.$ Area $_{\mathrm{DE}} /$ Area $\left._{\text {myocardium }}\right] \mathrm{x} 100 . \mathrm{LV}$ segmentation was performed according to the standardized 17 segment LV model $^{12}$. Each segment was graded for the presence DE on a 5 point scale proposed by Kim et al. (segmental scar score 0 - absence of DE; 1 - DE of 1-25\%; 2 - DE 26-50\%; 3 - DE 51-75\%; and 4 - DE 76-100\%) (ref. ${ }^{4}$ ). A segments with a scar score of 4 represented segments with totally transmural scar and non-viable myocardium. All examinations were performed and assessed by an experienced radiologist blinded to patient clinical data.

\section{Gated rest $\mathrm{Tc}^{99 \mathrm{~m}}$ sestamibi SPECT}

Gated SPECT imaging was performed using a 2-detector Siemens e.cam gamma camera (Siemens Medical Solutions, Erlangen, Germany) equipped with a low-energy, high-resolution parallel-hole collimators. Sixty-four projections were acquired in a $64 \times 64$ matrix over a $180^{\circ}$ from the $45^{\circ}$ right anterior oblique projection to the $45^{\circ}$ left posterior oblique projection. Images were gated at 8 frames per cardiac cycle. Processing was performed using a Butterworth filter with a frequency cut-off 0.45 cycles/ pixel and an order of 5.0. All patients underwent 1-day rest imaging with nitrate administration: $8 \mathrm{mCi}(296 \mathrm{MBq})$ $\mathrm{Tc}^{99 \mathrm{~m}}$ sestamibi (MIBI) was injected at rest, with SPECT imaging performed 1 hour after injection. Three hours later, $24 \mathrm{mCi}$ (888 MBq) $\mathrm{Tc}^{99 \mathrm{~m}} \mathrm{MIBI}$ was injected after sublingual nitrate administration $1 \mathrm{~h}$ before SPECT imaging. The data were analyzed visually and quantitatively. The segmental rest perfusion score was obtained through the use of the automated, commercially available software 4D-MSPECT (University of Michigan, Ann Arbor, USA). The tracer uptake was analyzed on computer-generated polar maps. In the perfusion analysis, the polar maps of each patient were compared on a pixel-by-pixel basis with the MIBI normal database derived from 70 normal volunteers. Pixels with MIBI activity greater than 2.5 SDs below the corresponding normal mean values were considered abnormal. A perfusion defect was automatically expressed by the computer as the number of abnormal pixels divided by the total number of LV pixels x 100 . Nonviable myocardial area was defined as that having MIBI uptake below 50\% of the maximum. Rest myocardial segmental perfusion was scored on a semi-quantitative five-point score scale of 0 to 4 ; where $0=$ normal, $1=$ equivocal, $2=$ moderate, $3=$ severe reduction of segment perfusion (radioisotope uptake) and $4=$ totally absent perfusion with absence of radioisotope uptake.

\section{Statistical analysis}

Descriptive statistics used for the analysis: $\mathrm{SL}_{\mathrm{Smax}}$ and DE were summarized as means and $95 \%$ confidence interval and medians with $5^{\text {th }}-95^{\text {th }}$ percentile range. $\mathrm{SL}_{\mathrm{Smax}}$, $\mathrm{DE}$ and $\mathrm{Q}_{\mathrm{REST}}$ in categories were described by their count and percentage. Inferential statistical tests were two-tailed, and all $P$ values of less than 0.05 were considered statisti- cally significant. To compare the STE and SPECT predictive accuracy, perfusion territories of the left anterior descending coronary artery (LAD), right coronary artery (RCA), and left circumflex artery (LCx) were evaluated separately. Segments 1, 2, 7, 8, 13, 14 were assigned to the LAD distribution. Segments 3, 4, 9, 10, and 15 were assigned to the RCA. Segments 5, 6, 11, 12, and 16 were assigned to the LCX distribution ${ }^{12}$.

Receiver operating curve (ROC) analysis was used to identify $\mathrm{SL}_{\mathrm{S} \max }$ optimal cut offs for DE categories. The results of the ROC analysis were described by area under curve (AUC), 95\% confidence interval and sensitivity and specificity at optimal cut off. These cut offs were used in further analyses. Individual endpoint of non-viable myocardium was defined according to $\mathrm{DE}>75 \%$ values and it was predicted with $\mathrm{SL}_{\text {Smax }}$ and $\mathrm{Q}_{\mathrm{REST}}$. Predictions were made with univariate models based on logistic regression first, and multivariate models were then used. Finally, the results from multivariate model were used in ROC analysis to prove the power of prediction. Predictions of DE using $\mathrm{Q}_{\mathrm{REST}}$ and $\mathrm{SL}_{\mathrm{Smax}}$ were compared by means of ROC analysis. Comparison of their predictive power to endpoint DE $>75 \%$ was computed using the method of DeLong et al. for the comparison of the ROC area under curve $^{13}$. Intraobserver and interobserver variabilities were reported using the Lin and Pearson correlation.

Analyses were computed using SPSS 18.0.3 (IBM Corporation, 2010) and MedCalc 11 (MedCalc Software 2010).

\section{RESULTS}

\section{Baseline study group characteristics}

Clinical baseline characteristics of the patients are given in Table 1 . Baseline heart rate and blood pressure were $78 \pm 8 / \mathrm{min}, 134 \pm 11 / 82 \pm 7 \mathrm{mmHg}$. The mean LV ejection fraction in the total study population was $34.3 \pm 4.8 \%$, the mean LV enddiastolic and endsystolic volume were $282 \pm 94$ resp. $184 \pm 67 \mathrm{~mL}$ respectively. Description of all parameters in the whole dataset and according to segments of LAD, LCX and RCA perfusion territory are clearly depicted in Tables 2 and 3.

In $1471 \mathrm{LV}$ segments (92\%) echocardiographic image quality allowed assessment of segmental function with STE compared with rest SPECT perfusion imaging in predicting transmural scar tissue on ceCMR (DE $>75 \%$ ). The distributions of DE and $\mathrm{SL}_{\mathrm{Smax}}$ in the whole study group according to segments assigned to the LAD, RCx a RCA perfusion territory are depicted in Fig. 1.

Among the 1471 segments with adequate image quality tracking of acoustic markers, segmental analysis of ceCMR indicated $0 \%$ DE in 725 segments, $1-25 \% \mathrm{DE}$ in 310 segments, $26-50 \%$ DE in 198 segments, $51-75 \%$ DE in 49 segments, and 76-100\% DE in 189 segments (non-viable myocardium). There were 1035 segments with 0-25\% DE (viable segments), 508 segments with 1-50\% DE (remote non-transmural myocardial infarcts) and 238 segments with DE 51-100\% (remote transmural myocardial infarcts). 

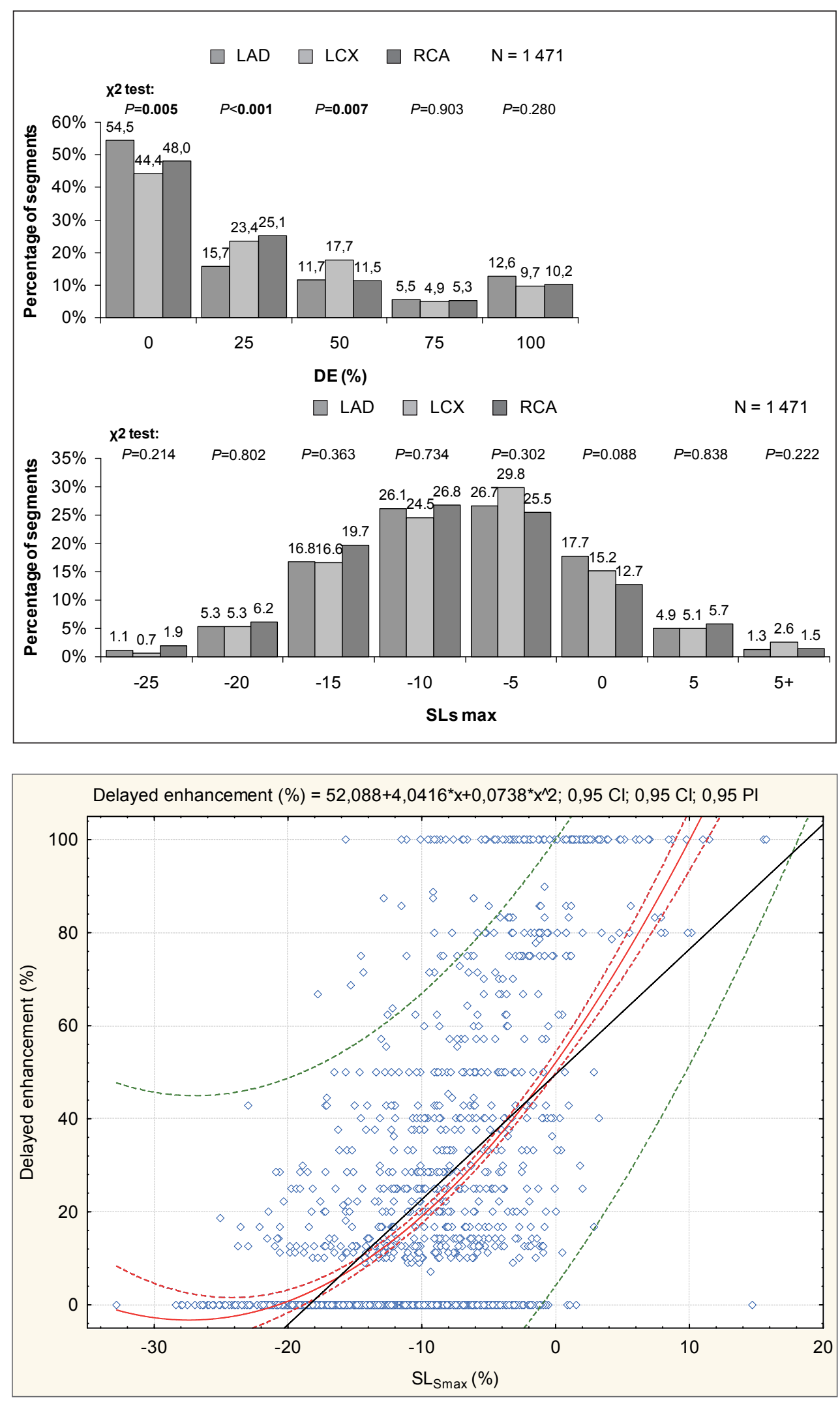

Fig. 1. Distribution of DE and $\mathrm{SL}_{\mathrm{Smax}}$ in the whole study group according to segments of LCx and RCA perfusion territory.

Fig. 2. Relationship between longitudinal peak systolic strain $\left(\mathrm{SL}_{\mathrm{Smax}}\right)$ and delayed enhancement (DE).

Segmental analysis of rest perfusion SPECT imaging indicated $Q_{\text {REST }} 0$ in 876 segments, $Q_{\text {REST }} 1$ in 123 segments, $Q_{\text {REST }} 2$ in 216 segments, $Q_{\text {REST }} 3$ in 164 segments, and $\mathrm{Q}_{\mathrm{REST}} 4$ in 92 segments.
Prediction of segmental non-viability using myocardial deformation and rest SPECT perfusion analysis in all segments and in individual coronary artery territories

A relationship existed between longitudinal left ventricular deformation and extent of scar tissue. $\mathrm{SL}_{\text {Smax }}$ had a significant correlation with the relative extent of $\mathrm{DE}$ (r=0.59, $P<0.0001$ ). Peak systolic strain increased with 
Table 1. Baseline study group characteristics.

\begin{tabular}{lc}
\hline Men/women & $86 / 14$ \\
Age (years) & $66 \pm 11$ \\
Height (cm) & $170 \pm 10$ \\
Weight (kg) & $87 \pm 13$ \\
History of myocardial infarction, n (\%) & $85(85 \%)$ \\
History of stroke, n (\%) & $19(19 \%)$ \\
Angina pectoris CCS class, (mean \pm SD) & $1.9 \pm 0,5$ \\
Ejection fraction, $\%$ & $34.3 \pm 4.8 \%$ \\
Heart failure NYHA class, n (\%) & \\
$\quad$ I & $19(19 \%)$ \\
II & $46(46 \%)$ \\
III & $35(35 \%)$ \\
IV & $0(0 \%)$ \\
Diabetes mellitus type 2, n (\%) & $42(42 \%)$ \\
Hypertension, n (\%) & $72(72 \%)$ \\
Dyslipidemia, n (\%) & $74(74 \%)$ \\
Smoking, n (\%) & $39(39 \%)$ \\
Coronary artery disease, n (\%) & \\
One-vessel disease & $5(5 \%)$ \\
Two-vessel disease & $25(25 \%)$ \\
Multi-vessel disease & $70(70 \%)$ \\
Left main coronary artery disease & $37(37 \%)$ \\
LAD disease & $90(90 \%)$ \\
Concomitant therapy, (\%) & \\
Betablockers & $84 \%$ \\
Calcium blockers & $93 \%$ \\
\hline
\end{tabular}

increasing scar transmurality. Scatter plot for correlation between $\mathrm{SL}_{\text {Smax }}$ and DE is depicted in Fig. 2. There was also significant correlation between extent of scar tissue (DE) and rest perfusion score $\left(\mathrm{Q}_{\mathrm{REST}}\right)(\mathrm{r}=0.6, P<0.0001)$. Correlation analysis showed that $\mathrm{SL}_{\mathrm{Smax}}$ also had a statistically significant relationship with the $\mathrm{Q}_{\mathrm{REST}}(\mathrm{r}=0.22$, $P<0.0001$ ).

The mean $\mathrm{SL}_{\text {Smax }}$ in segments with no scar (DE 0\%) was $-13.3 \pm 5.9 \%$, with LV myocardial scar and DE $1-25 \%$ $-10.3 \pm 5.2 \%$, DE $26-50 \%-7.9 \pm 5.2 \%$, DE $51-75 \%-6.4 \pm 4.1 \%$ and DE $76-100 \%-0.6 \pm 5.5 \%$.

Based on delayed enhancement findings by ceCMR, longitudinal strain analysis allowed distinction of segments with a transmural scar and totally non-viable myocardium ( $\mathrm{DE}>75 \%$ ) from segments with a non-transmural, potentially ischemic or morphologically normal myocardium. Performing ROC analysis for the distinction of transmural scarred non-viable myocardium from all other segments in the whole left ventricle, the area under the curve (AUC) was 0.866. The ROC analysis yielded a cut-off value of $-5.3 \%$ for $\mathrm{SL}_{\mathrm{Smax}}$ with a sensitivity of $83.1 \%$, specificity of $84.8 \%$ to identify segments with non-viable myocardium presence ( $\mathrm{DE}>75 \%$ ) on ceCMR. Odds ratios and ROC analysis results of given model with a $\mathrm{SL}_{\mathrm{Smax}}$ cutoff value $-5.3 \%$ for endpoint prediction ( $\mathrm{DE}>75 \%$ ) in all
Table 2. Description of parameters in the whole dataset.

\begin{tabular}{|c|c|c|}
\hline$n=1471$ & & Total $^{1}$ \\
\hline \multirow[t]{5}{*}{ DE (\%) } & Mean $(95 \% \mathrm{CI})$ & $22.5(20.9 ; 24.1)$ \\
\hline & $\begin{array}{c}\text { Median } \\
\left(5^{\text {th }} ; 95^{\text {th }} \text { perc. }\right)\end{array}$ & $10.0(0.0 ; 100.0)$ \\
\hline & $0 \%$ & $725(49.3 \%)$ \\
\hline & $\leq 25 \%$ & $979(66.6 \%)$ \\
\hline & $>75 \%$ & $189(12.8 \%)$ \\
\hline \multirow[t]{2}{*}{$\mathrm{SL}_{\mathrm{Smax}}$} & Mean $(95 \% \mathrm{CI})$ & $-10.0(-10.4 ;-9.7)$ \\
\hline & $\begin{array}{c}\text { Median } \\
\left(5^{\text {th }} ; 95^{\text {th }} \text { perc. }\right)\end{array}$ & $-10.1(-20.8 ; 1.2)$ \\
\hline SL $_{\text {Smax }}$ cut-off & $>-5.3$ & $189(12.8 \%)$ \\
\hline \multirow[t]{8}{*}{$\mathbf{Q}_{\text {REST }}$} & 0 & $876(59.6 \%)$ \\
\hline & 1 & $123(8.4 \%)$ \\
\hline & 2 & $216(14.7 \%)$ \\
\hline & 3 & $164(11.1 \%)$ \\
\hline & 4 & $92(6.3 \%)$ \\
\hline & $1-2$ & $339(23.0 \%)$ \\
\hline & $3-4$ & $256(17.4 \%)$ \\
\hline & $1-4$ & $595(40.4 \%)$ \\
\hline \multirow[t]{3}{*}{ Segments } & LAD & $547(37.2 \%)$ \\
\hline & $\mathrm{LCx}$ & $453(30.8 \%)$ \\
\hline & RCA & $471(32.0 \%)$ \\
\hline
\end{tabular}

${ }^{1}$ Categories of parameters as number and percentage of total, cut offs and categories are chosen according to definitions of endpoints used in the analysis

segments and in individual coronary arteries territories are depicted in Table 4.

To improve the accuracy of the model to predict an occurrence of non-viable myocardium, the optimum cutoff values $\mathrm{SL}_{\mathrm{S} \max }$ for segments corresponding to individual perfusion territories were subsequently identified $(-3.6 \%$, $-5.3 \%$ and $-4.7 \%$ for LAD, LCx and RCA perfusion territory). The results of ROC analysis corresponding to the optimum cut-off values derived for perfusion territories are given in Table 5.

$A Q_{\text {REST }}$ perfusion score 3-4 discriminated between segments with potentially viable myocardium and segments with $\mathrm{DE}>75 \%$ with a sensitivity of $86.2 \%$ and specificity of $66.3 \%$. The results of the segmental analyses in all left ventricular segments and in LAD, LCx and RCA perfusion territory segments using $\mathrm{Q}_{\mathrm{REST}}$ in prediction $\mathrm{DE} \geq 75 \%$ endpoint are presented in Tables 6 and 7.

Comparison of myocardial deformation and rest perfusion analysis in predicting transmural segmental non-viability

Odds ratios and ROC analysis results for prediction of non-viable myocardium ( $\mathrm{DE} \geq 75 \%$ endpoint) using $\mathrm{Q}_{\mathrm{REST}}$ and $\mathrm{SL}_{\mathrm{Smax}}$ are given in Table 6. Comparison of their predictive power to endpoint non-viable myocardium (DE $>75 \%$ ) in all left ventricular segments and in LAD, LCX 
Table 3. Description of parameters according to segments of LAD, LCx and RCA perfusion territory.

\begin{tabular}{|c|c|c|c|c|c|}
\hline \multirow{2}{*}{$\mathrm{n}=1471^{1}$} & & \multicolumn{4}{|c|}{ Segments } \\
\hline & & $\operatorname{LAD}(n=547)$ & $\operatorname{LCx}(n=453)$ & $\operatorname{RCA}(n=471)$ & $P$ value ${ }^{2}$ \\
\hline \multirow[t]{3}{*}{ DE (\%) } & Mean $(95 \% \mathrm{CI})$ & $22.7(19.9 ; 25.5)$ & $23.2(20.4 ; 26.0)$ & $21.5(18.8 ; 24.3)$ & - \\
\hline & Median $\left(5^{\text {th }} ; 95^{\text {th }}\right.$ perc. $)$ & $0.0(0.0 ; 100.0)$ & $12.5(0.0 ; 100.0)$ & $10.0(0.0 ; 100.0)$ & 0.090 \\
\hline & $>75 \%$ & $79(14.4 \%)$ & $52(11.5 \%)$ & $58(12.3 \%)$ & 0.346 \\
\hline \multirow[t]{2}{*}{$\mathrm{SL}_{\mathrm{Smax}}$} & Mean $(95 \% \mathrm{CI})$ & $-9.9(-10.5 ;-9.3)$ & $-9.5(-10.2 ;-8.9)$ & $-10.7(-11.3 ;-10.0)$ & - \\
\hline & Median $\left(5^{\text {th }} ; 95^{\text {th }}\right.$ perc. $)$ & $-9.9(-20.6 ; 0.8)$ & $-9.8(-20.7 ; 2.0)$ & $-10.8(-21.5 ; 1.2)$ & 0.042 \\
\hline \multirow[t]{3}{*}{$\mathrm{SL}_{\text {Smax }}$ cut-offs $^{3}$} & $>-5.3$ & - & $111(24.5 \%)$ & - & - \\
\hline & $>-4.7$ & - & - & $89(18.9 \%)$ & - \\
\hline & $>-3.6$ & $100(18.3 \%)$ & - & - & - \\
\hline \multirow[t]{8}{*}{$\mathbf{Q}_{\text {REST }}$} & 0 & $369(67.5 \%)$ & $254(56.1 \%)$ & $253(53.7 \%)$ & $<0.001$ \\
\hline & 1 & $38(6.9 \%)$ & $35(7.7 \%)$ & $50(10.6 \%)$ & 0.091 \\
\hline & 2 & $57(10.4 \%)$ & $69(15.2 \%)$ & $90(19.1 \%)$ & $<0.001$ \\
\hline & 3 & $55(10.1 \%)$ & $53(11.7 \%)$ & $56(11.9 \%)$ & 0.588 \\
\hline & 4 & $28(5.1 \%)$ & $42(9.3 \%)$ & $22(4.7 \%)$ & 0.006 \\
\hline & $1-2$ & $95(17.4 \%)$ & $104(23.0 \%)$ & $140(29.7 \%)$ & $<0.001$ \\
\hline & $3-4$ & $83(15.2 \%)$ & $95(21.0 \%)$ & $78(16.6 \%)$ & 0.047 \\
\hline & $1-4$ & $178(32.5 \%)$ & $199(43.9 \%)$ & $218(46.3 \%)$ & $<0.001$ \\
\hline
\end{tabular}

${ }^{1}$ Categories of parameters as number and percentage of total in given segment, cut offs and categories are chosen according to definitions of endpoints used in the analysis

${ }^{2}$ Statistical comparisons of segments were made with Kruskal-Wallis test for continuous parameters and with $\chi^{2}$ test for categorical parameters

${ }^{3}$ Optimal cut offs according to segments for DE $\geq 75 \%$ prediction

Table 4. Prediction of non-viable myocardium presence (DE $>75 \%$ endpoint) using a $\mathrm{SL}_{\text {Smax }}$ cut-off $>-5.3 \%$ in all left ventricular segments and in LAD, LCx and RCA perfusion territory segments.

\begin{tabular}{ccccc}
\hline & \multicolumn{5}{c}{ Endpoint DE $>$ 75\% } \\
\hline Cut-off $^{1}$ & All segments $\mathrm{n}=1471$ & LAD $\mathrm{n}=547$ & LCx $\mathrm{n}=453$ & RCA $\mathrm{n}=471$ \\
$\mathrm{SL}_{\mathrm{S} \max }>-5.3 \%$ & $27.00(17.93 ; 40.65)$ & $19.69(10.8 ; 35.9)$ & $27.42(12.4 ; 60.9)$ & $43.39(19.5 ; 96.7)$ \\
& $<0.001$ & $<0.001$ & $<0.001$ & $<0.001$ \\
\hline ROC AUC & 0.866 & 0.837 & 0.891 & 0.894 \\
$\mathbf{9 5 \%}$ CI $^{2}$ & $0.833 ; 0.900$ & $0.781 ; 0.892$ & $0.833 ; 0.949$ & $0.839 ; 0.949$ \\
$P^{2}$ & $<0.001$ & $<0.001$ & $<0.001$ & $<0.001$ \\
Sensitivity $^{3}$ & $83.1 \%$ & $79.7 \%$ & $84.6 \%$ & $86.2 \%$ \\
Specificity $^{3}$ & $84.6 \%$ & $83.3 \%$ & $83.3 \%$ & $87.4 \%$ \\
\hline
\end{tabular}

${ }^{1}$ Odds ratio for models based on logistic regression

${ }^{2}$ Area under curve based on ROC analysis supplemented by its $95 \%$ confidence interval and statistical significance

${ }^{3}$ Sensitivity and specificity of given model for endpoint prediction

and RCA perfusion territory segments was then calculated and results are depicted in Table 7. A minimum, but statistically significant difference (AUC 0.866 vs. 0.822 for $\mathrm{SL}_{\mathrm{Smax}}$ resp. $\mathrm{Q}_{\mathrm{REST}}, P=0.036$ ) in accuracy of predicting an occurrence of non-viable myocardium, as defined by $\mathrm{DE}>75 \%$ on ceCMR, was achieved as a result of higher accuracy of the deformation analysis in comparison with $\mathrm{Q}_{\text {REST }}$ in the RCA perfusion territory (AUC 0.75 vs. 0.893 for $\mathrm{Q}_{\mathrm{REST}}$ resp. $\mathrm{SL}_{\mathrm{Smax}}, P 0.001$ ).

\section{DISCUSSION}

The key conclusions of this study can be formulated as follows: 1) a cut-off value of $-5.3 \%$ for speckle tracking derived parameter $\mathrm{SL}_{\mathrm{Smax}}$ identified segments with nonviable myocardium presence ( $\mathrm{DE}>75 \%$ ) on ceCMR with a $83.1 \%$ sensitivity and $84.8 \%$ specificity; 2 ) the cut-off values $\mathrm{SL}_{\text {Smax }}$, which increase accuracy of the model predicting an occurrence of non-viable myocardium even more, can be derived for perfusion territories of individual coronary arteries corresponding approximately to the region of the LV frontal, lateral, and inferior myocardial wall; 3 ) as compared to the rest perfusion myocardial SPECT, 
Table 5. Identification of $\mathrm{SL}_{\mathrm{Smax}}$ optimal cut offs according to segments of $\mathrm{LAD}, \mathrm{LCx}$ and RCA perfusion territory.

\begin{tabular}{lccc}
\hline Endpoint; DE $>\mathbf{7 5 \%}{ }^{1}$ & LAD & LCx & RCA \\
\hline Risk group $(\mathrm{n} ; \%)^{2}$ & $79(14.4 \%)$ & $52(11.5 \%)$ & $58(12.3 \%)$ \\
AUC $^{3}$ & 0.896 & 0.904 & 0.945 \\
$95 \% \mathrm{CI}^{3}$ & $0.860 ; 0.932$ & $0.855 ; 0.953$ & $0.920 ; 0.971$ \\
$P^{4}$ & $<0.001$ & $<0.001$ & $<0.001$ \\
Cut-off $^{5}$ & -3.6 & -5.3 & -4.7 \\
Sensitivity $^{6}$ & $74.7 \%$ & $84.6 \%$ & $84.5 \%$ \\
Specificity $^{6}$ & $91.2 \%$ & $83.8 \%$ & $90.3 \%$ \\
\hline
\end{tabular}

${ }^{1}$ Non-viable myocardium

${ }^{2}$ Proportion of segments with given endpoint in analyzed group of segments

${ }^{3}$ Area under curve based on ROC analysis supplemented by its $95 \%$ confidence interval

${ }^{4}$ Statistical significance of AUC

${ }^{5}$ Optimal cut off of $\mathrm{SL}_{\text {Smax }}$ with the best combination of sensitivity and specificity

${ }^{6}$ Sensitivity and specificity at optimal cut off

Table 6. Prediction of non-viable myocardium ( $\mathrm{DE} \geq 75 \%$ endpoint) using $\mathrm{Q}_{\mathrm{REST}}$ and $\mathrm{SL}_{\mathrm{Smax}}$ in all left ventricular segments and in LAD, LCx and RCA perfusion territory segments.

\begin{tabular}{|c|c|c|c|c|c|c|}
\hline $\begin{array}{l}\text { Endpoint; } \\
\text { DE >75\% }\end{array}$ & Total & & LAD & & $\mathbf{L C x}$ & RCA \\
\hline \multicolumn{7}{|c|}{$\overline{\text { Univariate }^{2}-Q_{\text {REST }}}$} \\
\hline $\mathrm{Q}_{\mathrm{REST}} 0$ & $0.08(0.05 ; 0.12)$ & $<0.001$ & $0.04(0.02 ; 0.08)$ & $<0.001$ & $0.06(0.03 ; 0.17)<0.001$ & $0.19(0.10 ; 0.36)<0.001$ \\
\hline $\mathrm{Q}_{\mathrm{REST}} 1-2$ & $1.05(0.73 ; 1.50)$ & 0.789 & $2.09(1.20 ; 3.63)$ & 0.009 & $1.01(0.51 ; 2.00) \quad 0.983$ & $0.52(0.26 ; 1.03) \quad 0.059$ \\
\hline $\mathrm{Q}_{\mathrm{REST}} 3-4$ & $13.78(9.77 ; 19.42)$ & $<0.001$ & $19.16(10.86 ; 33.80)$ & $<0.001$ & $11.70(6.16 ; 22.22)<0.001$ & $13.09(7.09 ; 24.19)<0.001$ \\
\hline
\end{tabular}

\section{Multivariate $^{2,3}$}

$\mathrm{Q}_{\text {REST }} 0 \quad 0.20(0.12 ; 0.33)<0.001$

$\mathrm{Q}_{\text {REST }} 3-4 \quad 5.59(3.75 ; 8.32)<0.001 \quad 4.55(2.39 ; 8.68)<0.001 \quad 4.47(2.15 ; 9.30)<0.001 \quad 13.09(7.09 ; 24.19)<0.001$

Univariate $^{2}-\mathrm{SL}_{\mathrm{Smax}}$

$\mathrm{SL}_{\text {Smax }}{ }^{4} 27.00(17.93 ; 40.65)<0.001 \quad 30.72(16.86 ; 55.98)<0.001 \quad 27.34(12.31 ; 60.70)<0.001 \quad 50.77(23.23 ; 110.98)<0.001$ Multivariate $^{2}$

\begin{tabular}{llllll}
$\mathrm{SL}_{\mathrm{S} \max } 4,5$ & $14.11(9.06 ; 21.98)$ & $<0.001$ & $37.68(19.78 ; 71.78)<0.001$ & $14.22(5.51 ; 36.73)<0.001$ & $24.18(10.31 ; 56.70)<0.001$ \\
\hline
\end{tabular}

\footnotetext{
${ }^{1}$ Non-viable myocardium

${ }^{2}$ Odds ratio for univariate and multivariate models based on logistic regression; multivariate regression based on forward stepwise selection algorithm

${ }^{3}$ Only two $\mathrm{Q}_{\mathrm{REST}}$ categories, which were most significant in univariate model, are chosen into the multivariate model

${ }^{4}$ Cut-off for $\mathrm{SL}_{\text {Smax }}$ is used from previous table; patients above given cut-off are taken as "risk" group in the analysis (despite the value of OR for given endpoint)

${ }^{5}$ Multivariate regression model using different $\mathrm{SL}_{\mathrm{Smax}}$ cut-offs
}

the myocardium deformation analysis is more accurate in predicting LV non-viable myocardium, which is evident on the result obtained in the area of the inferior wall corresponding to the RCA myocardium perfusion territory.

\section{Determination of myocardial non-viability}

The assessment of myocardial viability might in future become a very important tool for preoperative risk stratification, since patients with pronounced LV systolic dysfunction have much increased perioperative risks and it would be desirable to identify subgroups of patients with the greatest potential benefits of revascularization. A meta-analysis showed that in patients with viability, subsequent revascularization was associated with $79.6 \%$ reduction in annual mortality, whereas the mortality of patients without viability after revascularization was much higher and not significantly different from medi- cally treated patients ${ }^{2}$. But the assessment of myocardial viability according to STICH (The Surgical Treatment for Ischemic Heart Failure) study protocol in patients with ischemic systolic dysfunction did not identify patients with a differential survival benefit from CABG, as compared with medical therapy alone ${ }^{14}$. Recently published data on patients with ischemic heart disease who underwent stress SPECT myocardial perfusion scintigraphy (MPS) suggest possible harm with revascularization in patients with extensive left ventricular scar (>10\% total myocardium fixed perfusion defect) especially if ischemia is not present ${ }^{15}$. These findings are consistent with the observation that cardiac stress PET imaging did not identify any therapeutic benefit in patients with ischemic left ventricular dysfunction (median LVEF 25\%) in the setting of observed extensive left ventricular scar (median LVEF $30 \%$ ) and little myocardial ischemia ${ }^{16}$. The above 
Table 7. Comparison of receiver operating curve analysis area under curves for predictions of non-viable myocardium (DE $>75 \%$ endpoint) using $\mathrm{Q}_{\mathrm{REST}}$ and $\mathrm{SL}_{\mathrm{Smax}}$.

\begin{tabular}{|c|c|c|c|c|}
\hline Endpoint; DE $\geq 75 \%^{5}$ & Total & LAD & $\mathbf{L C x}$ & RCA \\
\hline \multicolumn{5}{|l|}{$\mathrm{Q}_{\mathrm{REST}} \mathrm{ROC}^{1}$} \\
\hline $\mathrm{AUC}^{2}$ & 0.822 & 0.865 & 0.822 & 0.750 \\
\hline $95 \% \mathrm{CI}^{2}$ & $0.788 ; 0.855$ & $0.818 ; 0.911$ & $0.764 ; 0.881$ & $0.671 ; 0.828$ \\
\hline$P^{2}$ & $<0.001$ & $<0.001$ & $<0.001$ & $<0.001$ \\
\hline Sensitivity ${ }^{3}$ & $86.2 \%$ & $88.6 \%$ & $90.4 \%$ & $60.3 \%$ \\
\hline Specificity $^{3}$ & $66.3 \%$ & $76.9 \%$ & $62.1 \%$ & $89.6 \%$ \\
\hline \multicolumn{5}{|l|}{$\mathbf{S L}_{\text {Smax }}$ ROC $^{1}$} \\
\hline $\mathrm{AUC}^{2}$ & 0.866 & 0.840 & 0.891 & 0.893 \\
\hline $95 \% \mathrm{CI}^{2}$ & $0.833 ; 0.900$ & $0.780 ; 0.900$ & $0.833 ; 0.949$ & $0.836 ; 0.950$ \\
\hline$P^{2}$ & $<0.001$ & $<0.001$ & $<0.001$ & $<0.001$ \\
\hline Sensitivity $^{3}$ & $83.1 \%$ & $74.7 \%$ & $84.6 \%$ & $84.5 \%$ \\
\hline Specificity $^{3}$ & $84.6 \%$ & $91.2 \%$ & $83.3 \%$ & $90.3 \%$ \\
\hline \multicolumn{5}{|c|}{ Comparison of ROC AUC for $\mathbf{Q}_{\text {REST }}$ vs. $\mathrm{SL}_{\text {Smax }}$} \\
\hline$P^{4}$ & 0.036 & 0.458 & 0.100 & 0.001 \\
\hline
\end{tabular}

${ }^{1}$ ROC based on models based on logistic regression (from previous table)

${ }^{2}$ Area under curve based on ROC analysis supplemented by its $95 \%$ confidence interval and statistical significance

${ }^{3}$ Sensitivity and specificity of given model for endpoint prediction

${ }^{4}$ Statistical significance of comparison between AUC for multivariate model of $\mathrm{Q}_{\mathrm{REST}}$ and $\mathrm{AUC}$ for multivariate model of $\mathrm{SL}_{\mathrm{Smax}}$

${ }^{5}$ Nonviable myocardium

data indicate that the reverse remodeling with a positive influence on the prognosis and therapeutic benefit of revascularization, which significantly exceeds the periprocedural risk of revascularization, is feasible only in that percentage of patients having a minimum extent of the LV myocardial scar. This hypothesis is supported by the results of the previous study, which showed, that patients with 2 or less segments with the DE $>75 \%$ can be expected to show reverse LV remodeling with a sensitivity of $71 \%$ and specificity $67 \%\left(\right.$ ref. $\left.^{6}\right)$.

\section{Distinction between non-transmural and transmural infarction}

Available references provide no study results predicting extent of LV segment scar, as detected by the ceCMR with use of $\mathrm{DE}>75 \%$ phenomenon. In $\mathrm{DE}>50 \%$ segments, as stated by Kim et al. ${ }^{4}$, merely $12 \%$ show a probability of functional improvement after revascularization of segments with impaired contractility. Nevertheless, the data published later, Schwartzman et al. ${ }^{11}$, proved up to $35 \%$ of recoveries of regional contractility in originally dysfunctional segments with $\mathrm{DE}>50 \%$, which was the reason for our choosing the DE $>75 \%$ cut-off values, as a reliable marker for the occurrence of non-viable cicatrically-altered myocardium.

The deformation analysis of myocardium by means of evaluating two-dimensional strain was validated, both in an experimental and clinical model, as a relatively accurate and reproducible method in the differentiation between potentially viable myocardium with no or nontransmural scar tissue and chronic transmural myocardial infarct without the potential for functional recovery after revascularization ${ }^{7,17}$. Speckle tracking deformation analysis has increased sensitivity and specificity in comparison with tissue Doppler imaging for determining the transmural extent of a myocardial scar ${ }^{17}$. Becker et al. observed that radial strain predicted functional recovery after revascularization with an accuracy similar to that of ceCMR in patients with ischemic LV dysfunction'. Longitudinal strain parameters correlated with the degree of coronary artery stenosis, left ventricular (LV) ejection fraction (EF) and independently predicted LV remodeling following myocardial revascularization ${ }^{17-19}$. Roes et al. evaluated 90 patients with chronic ischemic LV dysfunction and reported that a regional longitudinal strain cutoff value of $-4.5 \%$ could distinguish a nontransmural scar from a transmural infarct (cut-off DE $>50 \%$ ) with sensitivity of $81.2 \%$ and specificity of $81.6 \%\left(\right.$ ref. $\left.^{10}\right)$.

\section{Deformation analysis and rest perfusion SPECT imaging in non-viable myocardium prediction in the current study \\ Compared to the results of the study by Roes et al. ${ }^{10}$, the longitudinal peak systolic strain parameter achieved a higher diagnostic accuracy in predicting non- viable myocardium, defined by the extent of myocardial scar LV $>75 \%$ DE with $83.1 \%$ sensitivity and $84.6 \%$ speci- ficity. The number of segments analyzed by deformation analysis was comparable in both studies (92\% our study, 93\% Roes et al.). Discrepancies in cut-off values derived for different endpoints ( $\mathrm{DE}>50 \%$ resp. $75 \%$ ) can be speculatively interpreted by the gravity of global systolic dysfunction of the examined individuals, and thus by a different extent of hibernating myocardium and ischemic LV dysfunction. All the patients in our study showed the}


baseline EF LV $\leq 40 \%$ (average EF LV $34 \pm 5 \%$ ), whereas Roes et al. presented the EF LV $28 \pm 8 \%$, which could indicate a different deformation capability of segments with a relatively comparable morphological impairment (scar extent), but a difference in the gravity of ischemia depending on the preexisting global function. Another possible explanation lies probably in a higher quantity of hibernating segments with ischemia at rest which determines not only the global function of myocardium (EF LV), but also a regional deformation of individual LV segments, independently of the scar extent, especially at hibernating viable LV segments. As the first paper published on this issue, our study has proven a higher accuracy in predicting a transmural myocardial scar, using cut-off specifically for segments of perfusion territories of individual coronary arteries.

Cardiac gated perfusion SPECT imaging is relatively widely available and very sensitive for detecting nonviable myocardium $^{5,15}$. Rest perfusion imaging score $3-4$, evaluated in a semi-quantitative scale by means of the gated SPECT myocardium, can be considered a correlate of non-viable myocardial tissue, and confirming a high rest summation score represents a strongly negative prognostic predictor ${ }^{5,15,20-22}$. While comparing the perfusion and deformation analysis, the myocardium SPECT statistically achieved, probably as a result of attenuation artifacts in the territory of the right coronary artery corresponding to the localization of the LV inferior wall, a significantly lower accuracy in predicting segments with transmural scar, in comparison with the deformation analysis (AUC 0.822 vs. $0.866, P=0.036$ ). So far this finding on the direct comparison between rest deformation analysis and perfusion of myocardium has not been published.

\section{Limitations}

The limitations of the present study included the lack of segmental function data and outcome after revascularization. Only $92 \%$ of segments with an adequate ultrasound quality for speckle tracking analysis were available for direct comparison with MR and SPECT. In order to definitely designate a significance of specifying viability of myocardium by means of the longitudinal rest deformation analysis and optimum management (revascularization vs. conservative therapy) of a patient with ischemic left ventricular systolic dysfunction, it will be indispensable to carry out a randomized prospective comparative study with a more strictly defined cut-off value that discriminates between viable (implying a profit from revascularization) and non-viable myocardium (conservative therapy), in comparison with the STICH study.

\section{CONCLUSIONS}

1. Myocardial deformation imaging based on 2-dimensional longitudinal peak systolic strain analysis could differentiate between viable myocardial tissue and a LV segment with transmural scar, as defined by DE > 75\% (which can be considered as a reliable marker of myocar- dium non-viability) in patients with ischemic left ventricular systolic dysfunction.

2 . The cut-off value of $-5.3 \%$ longitudinal peak systolic longitudinal 2D strain observed in the present study enabled the identification of non-viable segments with transmural scar tissue with a high accuracy demonstrating the potential use of longitudinal strain imaging for the selection of patients who will benefit from revascularization. For the first time, a definition was provided for cut-off values derived for perfusion territories of individual coronary arteries corresponding to myocardial segments of the frontal (LAD), lateral (LCX) and inferior walls (RCA), which further improve the prediction of transmural scars occurring in these regions.

3. This is the first study proving that the rest deformation analysis of myocardium is more accurate than that with perfusion myocardial SPECT, in predicting nonviable LV myocardium, which is derived from the result obtained in the area of the inferior wall corresponding to the RCA perfusion territory.

\section{CONFLICT OF INTEREST STATEMENT}

Author's conflict of interest disclosure: None declared.

\section{REFERENCES}

1. Gheorghiade M, Sopko G, De Luca L, Velazquez EJ, Parker JD, Binkley PF, Sadowski Z, Golba KS, Prior DL, Rouleau JL, Bonow RO. Navigating the Crossroads of Coronary Artery Disease and Heart Failure Circulation. Circulation 2006;114(11):1202-13.

2. Allman KC, Shaw LJ, Hachamovitch R, Udelson JE. Myocardial viability testing and impact of revascularization on prognosis in patients with coronary artery disease and left ventricular dysfunction: a meta-analysis. J Am Coll Cardiol 2002;39(7):1159-62.

3. Bax JJ, Poldermans D, Elhendy A, Cornel JH, Boersma E, Rambaldi R, Roelandt JR, Fioretti PM. Improvement of left ventricular ejection fraction, heart failure symptoms and prognosis after revascularization in patients with chronic coronary artery disease and viable myocardium detected by dobutamine stress echocardiography. J Am Coll Cardiol 1999;34:163-9.

4. Kim RJ, Wu E, Rafael A, Chen EL, Parker MA, Simonetti O, Klocke FJ, Bonow RO, Judd RM. The use of contrast-enhanced magnetic resonance imaging to identify reversible myocardial dysfunction. $\mathrm{N}$ Engl J Med 2000;343(20):1445-53.

5. Bax JJ, van der Wall EE, Harbinson M. Radionuclide techniques for the assessment of myocardial viability and hibernation. Heart 2004; 90(Suppl 5):26-33.

6. Skala T, Hutyra M, Vaclavik J, Kaminek M, Horak D, Novotny J, Zapletalova J, Lukl J, Marek D, Taborsky M. Prediction of long-term reverse left ventricular remodeling after revascularization or medical treatment in patients with ischemic cardiomyopathy: a comparative study between SPECT and MRI. Int J Cardiovasc Imaging 2011;27(3):343-53.

7. Amundsen $B H$, Helle-Valle $T$, Edvardsen $T$, Torp $H$, Crosby J, Lyseggen E, Støylen A, Ihlen H, Lima JA, Smiseth OA, Slørdahl SA. Noninvasive myocardial strain measurement by speckle tracking echocardiography: validation against sonomicrometry and tagged magnetic resonance imaging. J Am Coll Cardiol 2006;47(4):789-93.

8. Choi JO, Cho SW, Song YB, Cho SJ, Song BG, Lee SC, Park SW. Longitudinal $2 \mathrm{D}$ strain at rest predicts the presence of left main and three vessel coronary artery disease in patients without regional wall motion abnormality. Eur J Echocardiogr 2009;10(5):695-701.

9. Becker M, Lenzen A, Ocklenburg C, Stempel K, Kühl H, Neizel M, Katoh M, Kramann R, Wildberger J, Kelm M, Hoffmann R. Myocardial 
Deformation Imaging Based on Ultrasonic Pixel Tracking to Identify Reversible Myocardial Dysfunction. J Am Coll Cardiol 2008;51;147381.

10. Roes SD, Mollema SA, Lamb HJ, van der Wall EE, de Roos A, Bax JJ. Validation of echocardiographic two-dimensional speckle tracking longitudinal strain imaging for viability assessment in patients with chronic ischemic left ventricular dysfunction and comparison with contrast-enhanced magnetic resonance imaging. Am J Cardiol 2009;104(3):312-7.

11. Schvartzman PR, Srichai MB, Grimm RA, Obuchowski NA, Hammer DF, McCarthy PM, Kasper JM, White RD. Nonstress delayed enhancement magnetic resonance imaging of the myocardium predicts improvement of function after revascularization for chronic ischemic heart disease with left ventricular dysfunction. Am Heart J 2003;146:535-41.

12. Cerqueira MD, Weissman NJ, Dilsizian V, Jacobs AK, Kaul S, Laskey WK, Pennell DJ, Rumberger JA, Ryan T, Verani MS; American Heart Association Writing Group on Myocardial Segmentation and Registration for Cardiac Imaging. Standardized Myocardia Segmentation and Nomenclature for Tomographic Imaging of the Heart: A Statement for Healthcare Professionals From the Cardiac Imaging Committee of the Council on Clinical Cardiology of the American Heart Association. Circulation 2002;105:539-542

13. DeLong ER, DeLong DM, Clarke-Pearson DL. Comparing the areas under two or more correlated receiver operating characteristic curves: a nonparametric approach. Biometrics 1988,44,837-45.

14. Bonow RO, Maurer G, Lee KL, Holly TA, Binkley PF, Desvigne-Nickens P, Drozdz J, Farsky PS, Feldman AM, Doenst T, Michler RE, Berman DS, Nicolau JC, Pellikka PA, Wrobel K, Alotti N, Asch FM, Favaloro LE, She L, Velazquez EJ, Jones RH, Panza JA; STICH Trial Investigators. Myocardial viability and survival in ischemic left ventricular dysfunction. N Engl J Med 2011;364(17):1617-25.

15. Hachamovitch R, Rozanski A, Shaw LJ, Stone GW, Thomson LE, Friedman JD, Hayes SW, Cohen I, Germano G, Berman DS. Impact of ischaemia and scar on the therapeutic benefit derived from myocardial revascularization vs. medical therapy among patients undergoing stress-rest myocardial perfusion scintigraphy. Eur Heart J 2011;32(8):1012-24.

16. Tarakji KG, Brunken R, McCarthy PM, Al-Chekakie MO, Abdel-Latif A, Pothier CE, Blackstone EH, Lauer MS. Myocardial Viability Testing and the Effect of Early Intervention in Patients With Advanced Left Ventricular Systolic Dysfunction. Circulation 2006;113:230-7.

17. Geyer H, Caracciolo G, Abe H, Wilansky S, Carerj S, Gentile F, Nesser HJ, Khandheria B, Narula J, Sengupta PP. Assessment of Myocardial Mechanics Using Speckle Tracking Echocardiography: Fundamentals and Clinical Applications. J Am Soc Echocardiogr 2010;23:351-69.

18. Park YH, Kang SJ, Song JK, Lee EY, Song JM, Kang DH, Kim YH, Lee CW, Hong MK, Kim JJ, Park SW, Park SJ. Prognostic value of longitudinal strain after primary reperfusion therapy in patients with anterior-wall acute myocardial infarction. J Am Soc Echocardiogr 2008;21:262-7.

19. Delgado V, Mollema SA, Ypenburg C, Tops LF, van der Wall EE, Schalij MJ, Bax JJ. Relation between global left ventricular longitudinal strain assessed with novel automated function imaging and biplane left ventricular ejection fraction in patients with coronary artery disease. J Am Soc Echocardiogr 2008;21:1244-50.

20. Sciagra R, Leoncini L. Gated single-photon emission computed tomography. The present day "one-stop-shop" for cardiac imaging. $Q$ J Nucl Med 2005;49,19-29.

21. Hendel RC, Berman DS, Di Carli MF, Heidenreich PA, Henkin RE, Pellikka PA, Pohost GM, Williams KA; American College of Cardiology Foundation Appropriate Use Criteria Task Force; American Society of Nuclear Cardiology; American College of Radiology; American Heart Association; American Society of Echocardiography; Society of Cardiovascular Computed Tomography; Society for Cardiovascular Magnetic Resonance; Society of Nuclear Medicine. ACCF/ASNC/ ACR/AHA/ASE/SCCT/SCMR/SNM 2009 appropriate use criteria for cardiac radionuclide imaging: a report of the American College of Cardiology Foundation Appropriate Use Criteria Task Force, the American Society of Nuclear Cardiology, the American College of Radiology, the American Heart Association, the American Society of Echocardiography, the Society of Cardiovascular Computed Tomography, the Society for Cardiovascular Magnetic Resonance, and the Society of Nuclear Medicine. Circulation 2009;119(22):e561e587.

22. Berti V, Sciagrà R, Acampa W, Ricci F, Cerisano G, Gallicchio R, Vigorito C, Pupi A, Cuocolo A. Relationship between infarct size and severity measured by gated SPECT and long-term left ventricular remodelling after acute myocardial infarction. Eur J Nucl Med Mol Imaging 2011;38:1124-31 